\title{
An approach to the prediction of wax and asphaltene deposition in a pipeline based on Couette device experimental data
}

\author{
D. Eskin, J. Ratulowski, K. Akbarzadeh \& T. Lindvig \\ DBR Technology Center, Schlumberger, Canada
}

\begin{abstract}
An analysis of similarities of turbulent flows in a pipeline and a Couette device is performed. The transport processes in both cases are determined mainly by the boundary layer structure. A wax deposition model requires a single parameter to be determined from the Couette flow experiments. The asphaltene deposition modeling is more complicated and presented by the model framework only. The effect of the centrifugal force on asphaltene particle transport in a Couette device is investigated numerically. An approach to modeling wax deposition in a pipe based on Couette device experimental results is illustrated by a numerical example. The approaches developed can be straightforwardly applied for the transport pipeline design.
\end{abstract}

Keywords: asphaltene, Couette device, deposition, pipe, precipitation, scaling, turbulent transport, wax.

\section{Introduction}

Prevention of both wax and asphaltene deposition are important problems of oil transport in pipelines. The mechanisms of wax and asphaltene deposition are different. The major mechanism of wax deposition is a molecular diffusion. Due to intensive heat exchange between the outer pipeline wall and the cold environment (for example, sea water) the oil temperature in a pipeline wall vicinity may fall below the "wax appearance temperature" (WAT). Then, wax crystals precipitate from the fluid phase. On one hand the wax crystal concentration gradient directed towards the wall is highest near the wall where the temperature gradient oriented oppositely is biggest. On the other hand the wax particle concentration gradient causes the opposite gradient of wax 
molecules of the same absolute value. The latter causes the diffusion of wax molecules to the wall leading to the deposit layer formation. The growth of the deposit layer thickness is slowed down by the partial deposit removal caused by the shear flow in the vicinity of the deposit surface.

There are a number of papers on wax deposition modeling. Some models describing both the diffusion and the shear removal demonstrated fairly good performance (for example, [1]).

The physics of the asphaltene deposition phenomenon is fundamentally different from wax deposition. The asphaltene particles precipitate from oil when due to the friction losses the pressure in the pipeline drops below the "asphaltene precipitation onset pressure". Precipitated particles grow due to the molecular diffusion and the particle-particle aggregation, and eventually reach the wall forming the deposit layer. The asphaltene particles are usually characterized by a wide size distribution because they are prone to forming agglomerates, sizes of which may reach tens of microns. The major mechanisms of particle transport to the wall are (for example, [2]): 1) turbulent and Brownian diffusion; 2) turbophoresis. When a particle collides with the wall it sticks to the wall if the van der Waals attractive force is sufficient to prevent the particle removal by the shear flow. There are many papers devoted to modeling particle deposition in pipes. Most of them are concentrated on the particle transport to the wall only [2, 3]. There are no physically justified models for asphaltene deposition available in literature.

Since modeling both wax and asphaltene deposition is associated with a number of difficulties and uncertainties we employed a Couette Device for imitation of the complex deposition processes. In this device the inner cylinder is rotating while the outer one is immobile. The deposit layer is formed on the outer wall.

\section{Hydrodynamic similarity}

For providing similarity of the deposition process on the wall in a Couette device to that in a pipe the hydrodynamic conditions in the wall vicinity should be similar. For wax deposition the hydrodynamic similarity provides similarity of the shear removal process. For asphaltene deposition the hydrodynamic similarity guaranties the similarities of both particle transport and the probability of a particle sticking to the wall.

Note that in the case of wax deposition the temperature gradient at the wall should also be the same as that at the pipe wall.

The majority of transport pipelines operate under turbulent flow conditions. A Couette device imitating such a flow should also be run under similar turbulent conditions. Both pipe and Couette flows are relatively simple shear flows. The flow structure in such geometries can be considered as composed of a boundary layer flow and a core flow. Conventionally, the boundary layer is considered as consisting of a laminar sub-layer, a buffer layer and a turbulent boundary layer [4].

Within the laminar sub-layer the momentum transport is mainly controlled by the molecular viscosity. The thickness of this layer is evaluated as $\delta_{\mathrm{L}}=5 \delta^{+}$[4], 
where $\delta^{+}=v_{\mathrm{f}} / \mathrm{u}_{*}$ is the conventional non-dimensional thickness calculated assuming that the Reynolds number, based on the layer thickness and the velocity on its boundary, equals unity; $\mathrm{u}_{*}=\sqrt{\tau_{\mathrm{w}} / \rho_{\mathrm{f}}}$ is the friction velocity; $v_{\mathrm{f}}$ is the fluid kinematic viscosity; $\rho_{\mathrm{f}}$ is the fluid density; $\tau_{\mathrm{w}}$ is the shear stress at the wall. The velocity distribution within the laminar sub-layer is linear.

Within the buffer layer the momentum transport is controlled by both the molecular viscosity and the turbulence. The buffer layer thickness is usually evaluated as $\delta_{\mathrm{B}}=\mathrm{b} \delta^{+}$. Different authors use different values of the constant $\mathrm{b}$. Schlichting and Gersten [4] employed $b=65$. The velocity distribution within the buffer layer can be described by either a turbulence model or by a so called wall function. The wall function is a unique normalized velocity distribution valid for a wide range of flow parameters. The wall function for the buffer layer in a Couette device can be found in [4] formulated as $\mathrm{u}^{+}=\mathrm{f}\left(\mathrm{y}^{+}\right)$, where $\mathrm{u}^{+}=\mathrm{u} / \mathrm{u}_{*} ; \mathrm{u}$ is the circumferential flow velocity, $\mathrm{y}^{+}=\mathrm{y} / \delta^{+}$is the normalized coordinate; $y$ is the coordinate determining a position within the boundary layer ( $y=0$ at the wall). This normalized velocity distribution in the buffer layer can be employed for a pipe flow as well.

The momentum transport within the turbulent boundary layer is controlled by turbulence only. Maintaining high accuracy it can be assumed that the turbulent boundary layer extends to the channel center (this assumption is valid for both Couette and pipe flows). Then the velocity distribution in a core flow can be calculated by applying the Prandtl mixing length model. The velocity distribution in a pipe can be also accurately described by a power law function (see [4]).

As one can see from the above analysis the thicknesses of the laminar sublayer and the buffer layer are functions of the shear stress at the wall, $\tau_{\mathrm{w}}$, and the fluid viscosity, $v_{\mathrm{f}}$. Let us assume that the particle transport to the wall is not affected by inertial forces (particles are relatively small). Then the hydrodynamic similarity of two near-wall flows is obtained if the shear stress at the wall, $\tau_{\mathrm{w}}$, and the wall temperature, determining the fluid kinematic viscosity, $v_{f}$, are the same.

The shear stress at the pipe wall is calculated as [4]:

$$
\tau_{\mathrm{w}}=\rho_{\mathrm{f}} \mathrm{f} \frac{\mathrm{U}^{2}}{8}
$$

where $\mathrm{f}$ is the Fanning friction factor that is a function of the pipe Reynolds number and the surface roughness and $U$ is the superficial flow velocity.

Usually, the maximum roughness of the transport pipe walls is below $50 \mu \mathrm{m}$. Our calculations show that in the majority of flow regimes the transport pipelines are hydraulically smooth. Moreover, at the initial stage of the deposition process the cavities between asperities forming roughness are filled with deposit material, i.e., after a relatively short time the pipe surface is covered with 
deposit. A newly formed surface is hydraulically smooth therefore for calculation of the Fanning friction factor the Blausius correlation for a smooth pipe can be employed [4].

Based on the experimental particle velocity distribution in the Couette device buffer layer [4] and the velocity distribution in the core flow obtained on the basis of the Prandtl mixing length approach [4] we derived the analytical expression relating the non-dimensional torque $\mathrm{G}$ applied to the Couette device rotor and the Reynolds number $\operatorname{Re}_{\mathrm{c}}$ :

$$
\gamma(\eta) \frac{\operatorname{Re}_{c}}{\sqrt{G}}=\ln \sqrt{G}+\phi(\eta)+\xi
$$

where $\mathrm{G}=\mathrm{T} /\left(\rho_{\mathrm{f}} v_{\mathrm{f}}^{2} \mathrm{~L}\right)$ is the non-dimensional torque, $\mathrm{L}$ is the Couette device height, $\mathrm{r}_{0}, \mathrm{R}$ are the inner and outer radii of a Couette device respectively, $\operatorname{Re}_{\mathrm{c}}=\Omega \mathrm{r}_{0}\left(\mathrm{R}-\mathrm{r}_{0}\right) / \nu_{\mathrm{f}}$ is the Couette device Reynolds number, $\mathrm{T}=\tau_{\mathrm{w}} \cdot 2 \pi \mathrm{R}^{2} \mathrm{~L}$ is the torque, $\eta=r_{0} / R$ is the Couette device radius ratio, $\gamma=\frac{\kappa}{\frac{1}{\eta}+\eta} \frac{\sqrt{2 \pi}}{1-\eta}$, $\phi=\frac{2}{\frac{1}{\eta}+\eta}-\ln \left(\frac{1+\eta}{1-\eta}\right), \Omega$ is the inner cylinder angular velocity, $\xi=0.406$.

Equation (2) is in a good agreement with the experimental data for $\mathrm{Re}_{\mathrm{c}}>13000$ [5].

Thus, the rotation speed of a Couette device providing the shear stress at the outer wall that is equal to the stress at the pipe wall (Eq. (1)) is easily calculated by Eq. (2).

For asphaltene deposition it is important to consider the mechanism of a particle interaction with the wall. As it was mentioned above the main mechanisms of particle transport to the wall are turbulent and Brownian diffusions, and turbophoresis. Brownian diffusion is the dominating mechanism within the diffusive boundary layer only. The thickness of the diffusive layer can be evaluated by assuming that the Peclet number, calculated on the basis of the diffusive layer thickness and the velocity at the layer boundary, equals unity $\left(\mathrm{Pe}_{\mathrm{B}}=\mathrm{u}\left(\delta_{\mathrm{d}}\right) \delta_{\mathrm{d}} / \mathrm{D}_{\mathrm{B}}=1\right)$ :

$$
\delta_{\mathrm{d}} \sim \frac{\delta_{\mathrm{L}}}{\sqrt{\mathrm{Sc}_{\mathrm{B}}}}
$$

where $\mathrm{Sc}_{\mathrm{B}}=v_{f} / \mathrm{D}_{\mathrm{B}}$ is the Schmidt number, $\mathrm{D}_{\mathrm{B}}$ is the Brownian diffusivity.

Since for regular hydrocarbons and asphaltene particles the Schmidt numbers are usually very large (up to $10^{9}$ ) the diffusive boundary layer is much thinner than the hydrodynamic boundary layer. In the majority of practical cases the Brownian diffusion can be ignored.

The turbulent diffusion leads to the particle dispersion over a flow domain and plays an important role in particle transport. The turbulent diffusion decreases with increasing the particle size [2]. The turbophoresis is a 
phenomenon caused by the sharp decrease in turbulent kinetic energy in the wall vicinity. Due to this phenomenon particles acquire velocities directed to the wall. The turbophoretic effect increases with increasing the particle size [2].

Fortunately, as it will be shown below employing a Couette device for imitation of the deposition in a pipe allows simplified modeling the complicated transport processes.

\section{Influence of the centrifugal force on particle transport in a Couette device}

The centrifugal force in a Couette flow may lead to particle stratification in the Couette device, i.e., an uneven distribution of particles across the gap. This effect must be understood and quantified to properly imitate the deposition in a pipe by using a Couette device.

Let us calculate the particle concentration distribution in a turbulent Couette flow assuming the concentration distribution is steady-state. We will neglect the turbophoresis effect because 1) it reveals itself only in the wall vicinity and 2) it occurs in both pipe and Couette flows. Then the convection-diffusion equation takes the form:

$$
\mathrm{D}_{\mathrm{P}} \frac{\mathrm{d} \overline{\mathrm{c}}}{\mathrm{dr}}-\overline{\mathrm{c}} \mathrm{v}_{\mathrm{tr}}=0
$$

where $D_{p}$ is the particle diffusivity in a turbulent flow, $\bar{c}=c / c_{m} ; c_{m}$ is the mean particle concentration; $v_{t r}$ is the particle drift velocity in a fluid under action of the centrifugal force.

Since the amount of particles deposited is small the total volume of suspended particles in a Couette Device is assumed to be constant. Thus, the boundary condition for Eq. (4) is a volume conservation equation for solids:

$$
\int_{\mathrm{r}_{0}}^{\mathrm{R}} \overline{\mathrm{crdr}}=0.5\left(\mathrm{R}^{2}-\mathrm{r}_{0}^{2}\right)
$$

Thus, the distribution of the relative concentration $\overline{\mathrm{c}}$ does not depend on $\mathrm{c}_{\mathrm{m}}$.

Assuming that the particle circumferential velocity equals that of the fluid (the assumption is valid for relatively small particles) the particle drift velocity in radial direction can be evaluated by the Stokes settling equation in a centrifugal force field:

$$
\mathrm{v}_{\mathrm{tr}}=\frac{\mathrm{d}_{\mathrm{s}}^{2} \frac{\mathrm{u}(\mathrm{r})^{2}}{\mathrm{r}}\left(\rho_{\mathrm{s}}-\rho_{\mathrm{f}}\right)}{18 \mu_{\mathrm{f}}}
$$

where $d_{s}$ is the particle size, $\mu_{\mathrm{f}}$ is the fluid dynamic viscosity, $\rho_{\mathrm{s}}$ is the particle density.

Equation (6) was derived at ignoring the particle fluctuation velocity due to turbulence. However, it provides reasonable accuracy for relatively small particles, such as asphaltene particles, which do not exceed a few tens of 
micrometers. These are characterized by a relatively low density $\left(\rho_{\mathrm{s}} \approx 1200 \mathrm{~kg} / \mathrm{m}^{3}\right.$ ). Our evaluations show that the particle diffusivity $D_{p}$ in this case is close to the fluid turbulent diffusivity (the eddy diffusivity $D_{t}$ ). The latter is numerically close to the turbulent kinematic viscosity $v_{t}$ because the transport of momentum, mass and heat in a turbulent flow has the same mechanism [4]. Usually, it is assumed that $\mathrm{Sc}_{t}=v_{t} / \mathrm{D}_{\mathrm{t}}$ is in the range $0.8-1.0$. Note that the eddy and the turbulent thermal diffusivities are equal to each other.

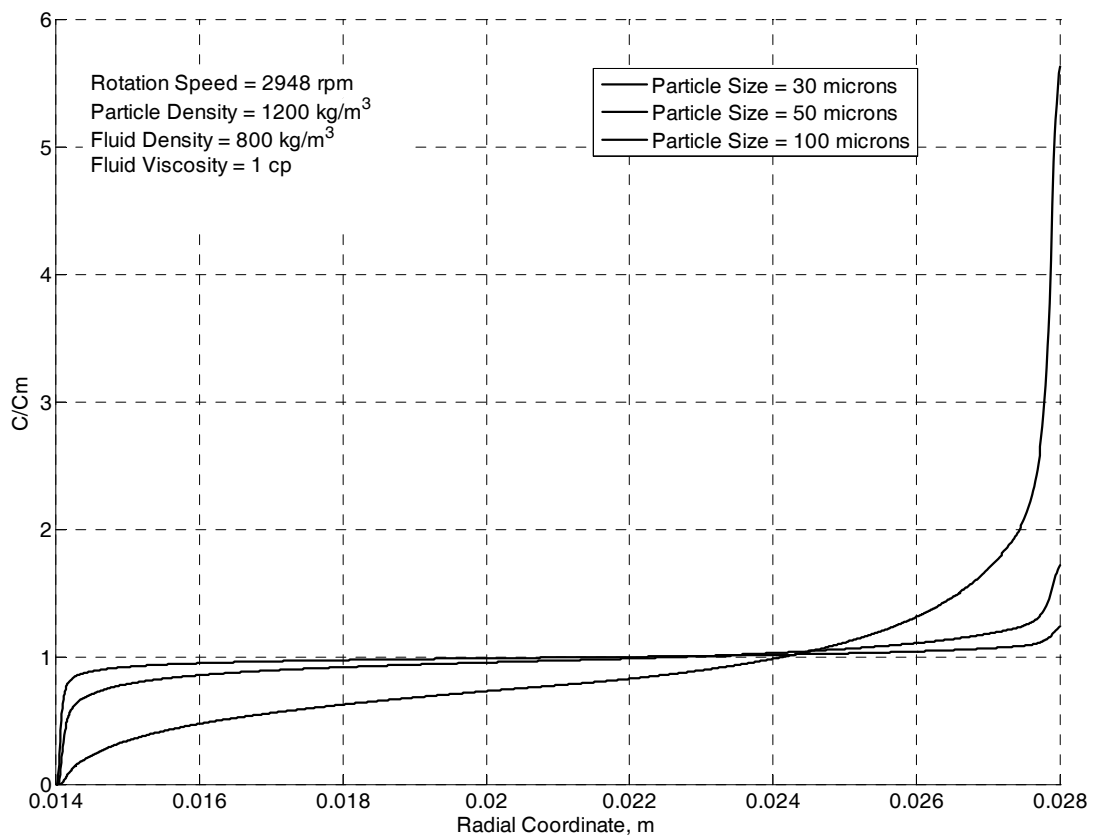

Figure 1: The normalized distributions of the volume concentration of asphaltene particles of different sizes along the radius of a Couette device.

Within the present research we will employ the empirical equation for the eddy diffusivity in the wall vicinity $\left(\mathrm{y}^{+} \leq 45\right)$ that can be found in [6]. The eddy diffusivity in the core flow can be calculated by the Prandtl mixing length model with reasonable accuracy (for example, [4]).

In Figure 1 we showed the normalized particle concentration distribution by volume vs. the Couette device radius calculated for the different particle sizes: $d_{s}=30,50$ and $100 \mu \mathrm{m}$. The dimensions of the Couette device were $\mathrm{r}_{0}=14 \mathrm{~mm}, \mathrm{R}=28 \mathrm{~mm}$ and $\mathrm{L}=70 \mathrm{~mm}$. The fluid viscosity was $\mu_{\mathrm{f}}=1 \mathrm{cp}$, the fluid density, $\rho_{\mathrm{f}}=800 \mathrm{~kg} / \mathrm{m}^{3}$. The particle density was 
$\rho_{\mathrm{s}}=1200 \mathrm{~kg} / \mathrm{m}^{3}$. The relatively high rotation speed $(\Omega=2948 \mathrm{rpm})$ was selected for calculations. One can see that the concentration distribution is relatively uniform for the $30 \mu \mathrm{m}$ particles, but an increase in the particle size leads to rapid strengthening of particle stratification.

Since we expect that not very big asphaltene particles (probably smaller than $20 \mu \mathrm{m}$ ) mainly contribute into the deposition there is a high possibility that the centrifugal stratification can be ignored in a deposition study by a Couette device.

\section{Calculation principles of the deposition process}

As it will be shown below employing a Couette device for deposition enables us to simplify modeling the complicated mechanisms of the particle transport and the deposit layer formation.

\subsection{Wax deposition calculation}

Let us characterize wax deposition in a pipeline of a given diameter $\mathrm{D}$ at the known superficial velocity $U$.

We employ a hypothesis that in the wall vicinity the precipitated particles are in thermodynamic equilibrium with the fluid. In this case the concentration distribution of precipitated wax particles near the wall is determined by the temperature distribution. Then the wax molecule flux to the wall can be evaluated as [1]:

$$
\mathrm{q}_{\mathrm{w}}=-\mathrm{D}_{\mathrm{m}}\left(\frac{\mathrm{dc}}{\mathrm{dr}}\right)_{\mathrm{r}=\mathrm{R}}=-\mathrm{D}_{\mathrm{m}}\left(\frac{\mathrm{dc}}{\mathrm{dT}}\right)_{\mathrm{T}=\mathrm{T}(\mathrm{R})}\left(\frac{\mathrm{dT}}{\mathrm{dr}}\right)_{\mathrm{r}=\mathrm{R}}
$$

where $D_{m}$ is the wax molecular diffusivity; $d c / d r$ is the gradient of the wax molecular concentration; $\mathrm{dc} / \mathrm{dT}$ is the rate of the wax molecular concentration change with temperature that can be measured or calculated.

Not the whole wax amount transported to the wall by the molecular diffusion will deposit due to shear removal. The removal rate depends on the shear stress at the wall and the rheological properties of the deposit layer. Currently, there is no clear understanding of mechanism of this phenomenon. The shear removal effect can approximately be taken into account by replacing the molecular diffusivity with an effective diffusivity, $\alpha$, that can be identified from a Couette device experiment. This approach is plausible since it is difficult to separately quantify the oil molecular diffusivity and the shear removal rate.

The wax flux contributing to the deposit growth is calculated as:

$$
\mathrm{q}_{\mathrm{d}}=-\alpha\left(\frac{\mathrm{dc}}{\mathrm{dr}}\right)_{\mathrm{r}=\mathrm{R}}=-\alpha\left(\frac{\mathrm{dc}}{\mathrm{dT}}\right)_{\mathrm{T}=\mathrm{T}(\mathrm{R})}\left(\frac{\mathrm{dT}}{\mathrm{dr}}\right)_{\mathrm{r}=\mathrm{R}}
$$

where $\alpha$ is the effective diffusivity of wax molecules.

Note that for this application the Couette device is equipped with a heater incorporated into the inner cylinder, and a cooling jacket mounted over the outer 
cylinder. This design allows maintaining the temperature of the outer wall constant and below WAT as well as controlling the temperature gradient at the wall to imitate deposition conditions in a pipe.

The amount of wax deposited in the Couette device during the time $t$, at neglecting the wax depletion effect, is calculated as:

$$
M_{d}=q_{d} \rho_{s} 2 \pi R L \cdot t=-\alpha\left(\frac{d c}{d T}\right)_{T=T(R)}\left(\frac{d T}{d r}\right)_{r=R} \rho_{s} 2 \pi R L \cdot t
$$

Thus, if the deposit amount in a Couette device is accurately measured, the effective diffusivity $\alpha$ can be straightforwardly calculated from Eq. (9).

Let us illustrate how this technique can be applied for calculating the deposition layer thickness in pipe.

To determine $\alpha$ we need to run a Couette device experiment. For providing the shear stress at the outer wall equal to that at the pipe wall Eqs. (1) and (2) have to be used to calculate the required inner cylinder rotation speed. For providing the equality of the temperature gradients at equivalent hydrodynamic conditions in the wall vicinity the heat flux through the wall of the Couette device should be equal to that through the pipe wall.

The heat balance equation for pipe flow can be written as follows:

$$
\frac{\mathrm{dT}_{\mathrm{b}}}{\mathrm{dx}}=-\frac{4 \mathrm{k}_{\mathrm{bw}}\left(\mathrm{T}_{\mathrm{b}}-\mathrm{T}_{\mathrm{w}}\right)}{\rho_{\mathrm{f}} \mathrm{c}_{\mathrm{p}} \mathrm{UD}}
$$

where $c_{p}$ is the isobaric heat capacity of a fluid, D is the pipe diameter, $k_{b w}$ is the heat transfer coefficient from the fluid to the pipe wall, $T_{b}$ is the temperature in the central area of a pipe, $\mathrm{T}_{\mathrm{w}}$ is the temperature at the deposit surface (initially, at the wall surface), $\mathrm{x}$ is the coordinate along a pipeline.

The temperature at the deposit surface is below the wax appearance temperature. The initial temperatures $\mathrm{T}_{\mathrm{b} 0}$ and $\mathrm{T}_{\mathrm{w} 0}$ at $\mathrm{x}=\mathrm{x}_{0}$ are given.

For the illustrative purpose only we use a simplified approach. To avoid the routine calculation of the heat transfer through the growing deposit layer we assume that the temperature at the deposit layer surface $T_{w}$ is constant along a pipe. This assumption to some extent takes into account an observation that the low conductivity of the growing deposit layer prevents the deposit surface from cooling. The same assumption means also that the deposit layer should be relatively thin and we can ignore an increase in the temperature $T_{w}$ at a fixed pipe cross-section in time due to the deposit layer insulation effect. Then we can also assume that the physical parameters of a fluid remain constant along a pipeline. Thus, the heat transfer coefficient $\mathrm{k}_{\mathrm{bw}}$ is constant. Then Eq. (10) is solved analytically. After performing a routine math we obtain the distribution of the temperature difference $\Delta \mathrm{T}=\mathrm{T}_{\mathrm{b}}-\mathrm{T}_{\mathrm{w}}$ along a pipe as:

$$
\Delta \mathrm{T}=\Delta \mathrm{T}_{0} \mathrm{e}^{-\frac{\mathrm{Nu}}{\operatorname{Re} \operatorname{Pr} \frac{\mathrm{x}}{\mathrm{D}}}}
$$


where $\mathrm{Nu}=\mathrm{k}_{\mathrm{bw}} \mathrm{D} / \lambda_{\mathrm{f}}$ is the Nusselt number, $\lambda_{\mathrm{f}}$ is the fluid heat conductivity, $\operatorname{Pr}=v_{\mathrm{f}} / \mathrm{a}_{\mathrm{f}}$ is the Prandtl number, $\mathrm{a}_{\mathrm{f}}=\rho_{\mathrm{f}} \mathrm{c}_{\mathrm{p}} / \lambda_{\mathrm{f}}$ is the fluid thermal diffusivity.

The Nusselt number can be evaluated by the empirical equation as follows [7]:

$$
\mathrm{Nu}=0.027 \mathrm{Re}^{0.8} \operatorname{Pr}^{0.33}
$$

The temperature gradient at the wall is calculated as:

$$
\left(\frac{\mathrm{dT}}{\mathrm{dr}}\right)_{\mathrm{r}=\mathrm{R}}=-\frac{\mathrm{q}_{\mathrm{bw}}}{\lambda_{\mathrm{f}}}=-\frac{\mathrm{k}_{\mathrm{bw}} \Delta \mathrm{T}}{\lambda_{\mathrm{f}}}
$$

where $\mathrm{q}_{\mathrm{bw}}$ is the heat flux to the wall.

Substituting this equation into Eq. (9), we get the following deposit flux to the wall:

$$
\mathrm{q}_{\mathrm{d}}(\mathrm{x})=-\alpha\left(\frac{\mathrm{dc}}{\mathrm{dr}}\right)_{\mathrm{r}=\mathrm{R}}=\alpha \frac{\mathrm{k}_{\mathrm{bw}}}{\lambda_{\mathrm{f}}}\left(\frac{\mathrm{dc}}{\mathrm{dT}}\right)_{\mathrm{T}=\mathrm{T}(\mathrm{R})} \Delta \mathrm{T}_{0} \mathrm{e}^{-\frac{\mathrm{Nu}}{\operatorname{Re} \operatorname{Pr} \frac{\mathrm{x}}{\mathrm{D}}}}
$$

The deposit layer thickness is calculated from the deposit volume balance as:

$$
\delta(\mathrm{x})=\frac{\mathrm{q}_{\mathrm{d}}(\mathrm{x}) \cdot \mathrm{t}}{1-\varphi}
$$

where $t$ is the time, $\varphi$ is the wax deposit layer porosity.

\subsubsection{Calculation examples}

Let us now consider a pipeline with diameter $\mathrm{D}=0.1778 \mathrm{~m}$ and length $\mathrm{L}_{\mathrm{p}}=3200 \mathrm{~m}$ in which oil of a certain chemical composition flows at a superficial velocity $U=3.1 \mathrm{~m} / \mathrm{s}$. The initial oil temperature is set to $\mathrm{T}_{\mathrm{b}}=350 \mathrm{~K}$ and the wall temperature $\mathrm{T}_{\mathrm{w}}=322 \mathrm{~K}$. The oil dynamic viscosity is assumed to be $\mu_{\mathrm{f}}=7 \cdot 10^{-3} \mathrm{~Pa} \cdot \mathrm{s}$, the density $\rho_{\mathrm{f}}=843 \mathrm{~kg} / \mathrm{m}^{3}$, the heat conductivity $\lambda_{\mathrm{f}}=0.15 \mathrm{~W} /(\mathrm{m} \cdot \mathrm{K})$, the heat capacity $\mathrm{c}_{\mathrm{p}}=2020 \mathrm{~J} /(\mathrm{kg} \cdot \mathrm{K})$. The rate of the wax molecular concentration change with temperature at the wall, calculated for the known oil chemical composition by the DBRSolids commercial software, is $(\mathrm{dc} / \mathrm{dT})_{\mathrm{T}=322 \mathrm{~K}}=1.28 \cdot 10^{-4} 1 / \mathrm{K}$. The wax particle density is $\rho_{\mathrm{s}}=900 \mathrm{~kg} / \mathrm{m}^{3}$. The deposition experiment in the Couette device (the dimensions were presented above) was conducted at a rotation speed of the inner cylinder set to $\Omega=3900 \mathrm{rpm}$. The wall temperature and the temperature gradient were maintained the same as those in the pipeline. The two hour experiment produced $\mathrm{M}=270 \mathrm{mg}$ of wax deposited on the outer wall. The effective diffusivity, calculated by Eq. (9) was $\alpha=1.66 \cdot 10^{-10} \mathrm{~m}^{2} / \mathrm{s}$ that is in line with the literature data on the molecular diffusivity [1].

In Fig. 2 we showed the calculated thicknesses of the wax deposit obtained at various flow times: $\mathrm{t}=10,50,100 \mathrm{~h}$. The wax deposit thickness linearly 
increases with time (Eq. (14)) and sharply decreases along the pipeline due to rapid reduction of the temperature gradient at the wall caused by fluid cooling. Note that the effect of the deposit layer growth rate reduction in time due to the deposit insulation effect is not taken into account by the employed simplified model.

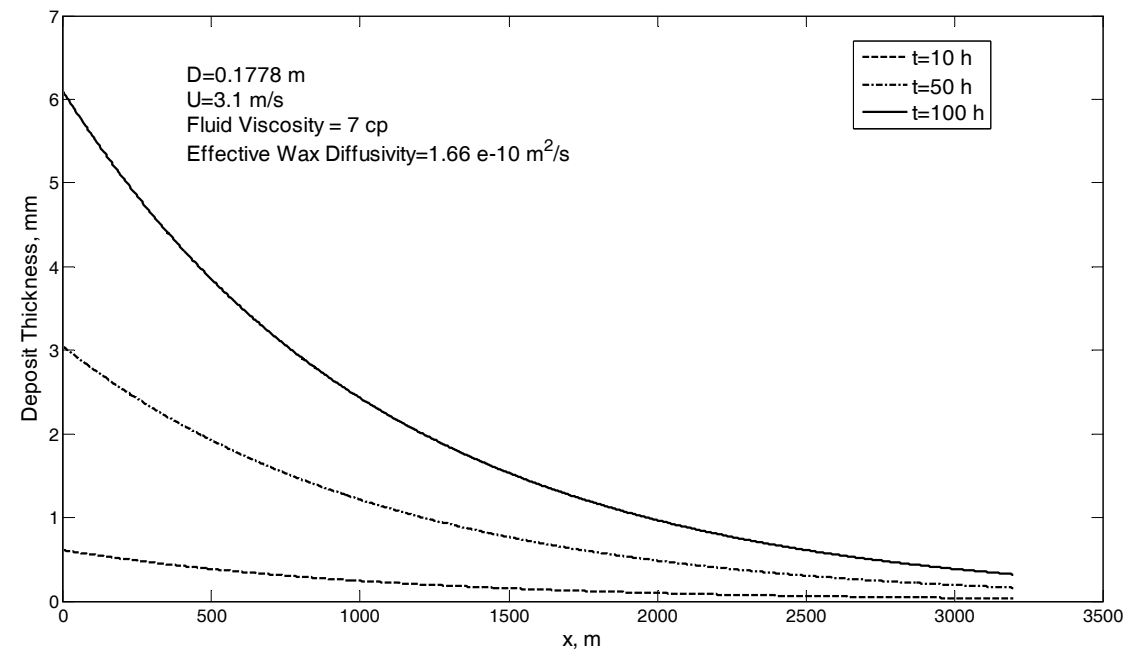

Figure 2: Distribution of the deposit thickness along a pipeline for the different operation times.

\subsection{Asphaltene deposition modeling}

The forecasting of asphaltene deposition is a more complicated problem than that of wax deposition. In this paper we present the model framework only.

Guha [2] suggested a robust convection-diffusion model, according to which the volume flux of particles depositing on the wall is determined as:

$$
J=-\left(D_{t}+D_{B}\right) \frac{d c}{d y}+c V_{p y}
$$

where $V_{p y}$ is the particle drift velocity caused by turbophoresis, that can be calculated for a given particle size [2].

The first right-hand side term determines the particle flux due to Brownian and turbulent diffusion, the second term due to the turbophoresis.

Equation (15) requires a boundary condition at the wall that should be set in dependence on the probability of a single particle deposition after a contact with the wall. Note that no clear approach to the boundary condition formulation is available in the literature.

The same author [2] showed that the deposition mechanism (diffusion or turbophoresis) is determined by the particle inertia. The particle velocity relaxation time $\tau=\rho_{\mathrm{s}} \mathrm{d}_{\mathrm{s}}^{2} /\left(18 \mu_{\mathrm{f}}\right)$ is employed as the measure of the inertia. It is 
convenient to use the dimensionless particle relaxation time: $\tau^{+}=\tau \mathrm{u}_{*}^{2} / v_{\mathrm{f}}$. Guha [2] compared the deposition rate results obtained by the convection-diffusion model with the experimental data for deposition of aerosol droplets in a pipe. It was assumed in the calculations that all particles reaching the wall deposit on it. The author [2] demonstrated that small particles $\left(\tau^{+}<\approx 0.1\right)$ move to the wall mainly due to the diffusion, whereas for large particles $\left(\tau^{+}>\approx 1\right)$ turbophoresis dominates. Thus, there is a significant size range where both diffusion and turbophoresis are important. It is should be noted that according to Guha [2] in the medium particle size range $\left(0.1 \approx<\tau_{+}<\approx 1\right)$ the calculated results deviate noticeably from the experimental data.

An interpretation of the Couette device experimental data for asphaltene deposition is complicated because both the particle size distribution and the deposition mechanism are not known a priori. Let us assume that only particles smaller than a certain (critical) size $d_{c r}$ stick to the wall as a result of a particlewall collision. This assumption is explained by considering the force balance for a particle attached to the wall. A drag force tending to remove the particle from the wall increases with increasing the particle size, while the particle - wall van der Waals attraction force per unit mass is reduced. The flux of "small" particles to the wall can be determined as (see Eq. (15)):

$$
J_{d}=-D_{p}\left(\frac{d c^{\text {small }}}{d y}\right)_{y=5 \delta^{+}}+\left(c^{\text {small }} V_{p y}^{\text {small }}\right)_{y=5 \delta_{+}}
$$

where $c^{\text {small }}$ is the volume concentration of "small" particles, i.e. particles smaller than the critical size $d_{c r}, V_{p y}^{\text {small }}$ is the mean turbophoretic velocity of small particles.

For the sake of convenience we consider the particle flux at the laminar boundary sub-layer surface $\left(\mathrm{y}^{+}=5\right)$. This flux is practically equal to the flux of particles depositing on the wall under steady-state conditions (the continuity equation for particles within the boundary layer is $\mathrm{dJ}_{\mathrm{d}} / \mathrm{dy}=0$ ). Since the particles are small the particle diffusivity is approximately equal to the eddy diffusivity $\left(D_{p}=D_{t}\right)$. According to Notter and Sleicher [6] the eddy diffusivity at the laminar sub-layer surface is: $\mathrm{D}_{\mathrm{t}}\left(\mathrm{y}^{+}=5\right)=0.104 \cdot v_{\mathrm{f}}$.

Assuming that the rate of establishing the concentration distribution profile along a Couette device radius is much higher than the rate of changing the concentration of small particles in the Couette device, we obtain an equation that describes the evolution of the mean concentration of small particles in time:

$$
\frac{d c_{m}^{\text {small }}(t)}{d t}=q_{v}^{\text {small }}(t)-J_{d} \frac{2 \pi R L}{V_{c}}
$$

where $\mathrm{q}_{\mathrm{v}}^{\text {small }}$ is the volumetric rate of generation of small particles, $\mathrm{V}_{\mathrm{c}}$ is the volume of a Couette chamber. 
On the basis of Eqs. (17) and (16) we formulate the convection-diffusion equation describing the deposition process:

$$
\begin{aligned}
\frac{\mathrm{dc}_{\mathrm{m}}^{\text {small }}}{\mathrm{dt}} & =\mathrm{q}_{\mathrm{v}}^{\text {small }}(\mathrm{t})-\mathrm{c}_{\mathrm{m}}^{\text {small }}\left(0.104 v_{\mathrm{f}}\left(\frac{\mathrm{d} \overline{\mathrm{c}}^{\text {small }}}{\mathrm{dy}}\right)_{\mathrm{y}=5 \delta^{+}}+\left(\overline{\mathrm{c}}^{\text {small }} \mathrm{V}_{\mathrm{py}}^{\text {small }}\right)_{\mathrm{y}=5 \delta_{+}}\right) \frac{2}{\mathrm{R}\left(1-\eta^{2}\right)}= \\
& =\mathrm{q}_{\mathrm{v}}^{\text {small }}(\mathrm{t})-\mathrm{c}_{\mathrm{m}}^{\text {small }} \beta \frac{2}{\mathrm{R}\left(1-\eta^{2}\right)}
\end{aligned}
$$

where $\overline{\mathrm{c}}^{\text {small }}=\mathrm{c}^{\text {small }} / \mathrm{c}_{\mathrm{m}}^{\text {small }}$ is the relative volume concentration of small particles, $\beta$ is the effective deposition velocity, which is constant for given flow parameters.

The initial condition for this equation is that the initial volume concentration of small particles is zero: $\mathrm{c}_{\mathrm{m}}^{\text {small }}(0)=0$.

Since the parameter $\beta$ does not depend on the concentration it can be identified from a constant pressure Couette device experiment where asphaltene particles are pre-generated. The particle generation term in Eq. (18) is zero in this case. Assuming that the initial condition is $\mathrm{c}_{\mathrm{m}}^{\text {small }}(0)=\mathrm{c}_{\mathrm{m} 0}^{\text {small }}$ we obtain the analytical solution as:

$$
c_{m}^{\text {small }}(t)=c_{m 0}^{\text {small }} e^{-\frac{2 \beta t}{R\left(1-\eta^{2}\right)}}
$$

The deposit mass for the time $t$ is then calculated as:

$$
M(t)=\rho_{s} 2 \pi R L \cdot \beta \int_{0}^{t} c_{m}^{\text {small }} d t=\rho_{s} \pi R^{2}\left(1-\eta^{2}\right) L \cdot c_{m 0}^{\text {small }}\left(1-e^{-\frac{2 \beta t}{R\left(1-\eta^{2}\right)}}\right)
$$

There are two unknowns in this equation: $\beta$ and $\mathrm{c}_{\mathrm{m} 0}^{\text {small }}$. To exclude the variable $\mathrm{c}_{\mathrm{m} 0}^{\text {small }}$ we can use the results of two deposition experiments of two different durations $\left(t_{1}\right.$ and $\left.t_{2}\right)$. Then the effective deposition velocity can be determined from the following equation:

$$
\frac{M\left(t_{1}\right)}{M\left(t_{2}\right)}=\frac{1-e^{-\frac{2 \beta t_{1}}{R\left(1-\eta^{2}\right)}}}{1-e^{-\frac{2 \beta t_{2}}{R\left(1-\eta^{2}\right)}}}
$$

One of the most difficult problems is evaluating the rate of small particle generation. It is expected that this generation rate should change from maximum to zero due to the fluid depletion. The evaluation of this rate is possible if monitor the deposit mass while conducting the deposition experiments at a gradual pressure reduction with the same rate as that observed in the pipeline $(\mathrm{dp} / \mathrm{dt}=\mathrm{Udp} / \mathrm{dx})$.

The effective deposition velocity and the rate of small particle generation determined by the Couette device experiment can be straightforwardly employed for predicting the asphaltene deposit thickness in a pipeline. 


\section{Conclusions}

An analysis of similarities of turbulent flows in a Pipeline and a Couette device has been performed. It has been shown that the transport processes in both cases are determined mainly by the boundary layer structure. The wax deposition model requires a single parameter to be determined from the Couette experiments. The asphaltene deposition modeling is more complicated and only the model framework has been presented here. The effect of the centrifugal force on asphaltene particle transport in a Couette device has been investigated numerically. It has been also demonstrated how experimental data obtained in the Couette device can be used to predict the wax deposition thickness along a pipeline at different production times.

\section{References}

[1] Akbarzadeh K., Zougari M., Introduction to a Novel Approach for Modeling Wax Deposition in Fluid Flows. 1. Taylor-Couette System Ind. Eng. Chem. Res., 47(3), pp 953-963, 2008.

[2] Guha A, Transport and Deposition of Particles in Turbulent and Laminar Flow, Annual Review of Fluid Mechanics, 40, pp. 311-341, 2008.

[3] Johansen S.T., The Deposition of Particles on Vertical Walls, International Journal of Multiphase Flow, 17(3), pp. 355-376, 1991.

[4] Schlichting H., Gersten K., Boundary-Layer Theory, Springer-Verlag, Berlin, Heidelberg, New York, 2000.

[5] Lewis G.S. and Swinney H.L., Velocity Structure Functions, Scaling and Transitions in High-Reynolds-Number Couette-Taylor Flow, Physical Review, 59(5), pp. 5457-5467, 1999

[6] Notter R.H., Sleicher C.A., Eddy Diffusivity in the Turbulent Boundary Layer Near a Wall, Chemical Engineering Science, 26(1), pp. 161-171, 1971.

[7] Rohsenow W.M., Hartnett J. and Cho Y.I., Handbook of Heat Transfer, McGraw-Hill, 3-rd ed., 1998. 